\title{
Distinguishing Schrödinger cats in a lossy environment
}

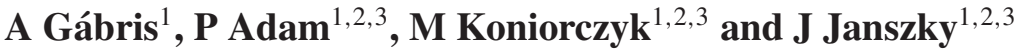 \\ ${ }^{1}$ Research Institute of Solid State Physics and Optics, Hungarian Academy of Sciences, \\ H-1525 Budapest, PO Box 49, Hungary \\ ${ }^{2}$ Research Group for Nonlinear Optics, Hungarian Academy of Sciences, Hungary \\ ${ }^{3}$ Institute of Physics, University of Pécs, Ifjúság út 6, H-7624 Pécs, Hungary
}

Received 4 July 2003, accepted for publication 16 December 2003

Published 5 March 2004

Online at stacks.iop.org/JOptB/6/S84 (DOI: 10.1088/1464-4266/6/3/014)

\begin{abstract}
Optical Schrödinger cat states - that is, even and odd coherent states-are considered as possible candidates for forming a computational basis for a coherent state qubit. The distinguishability of the two originally orthogonal states after experiencing loss is quantified in terms of quantum relative entropy. This is a physically instructive quantity related to probabilities of faults in identifying the state. This distinguishability is important for classical communication and for the problem of reading out the result of a quantum computation by a lossy device. It is shown that the distinguishability can significantly increase if the environment is prepared in an appropriately chosen squeezed state.
\end{abstract}

Keywords: coherent state superposition, optical qubit, squeezed state, decoherence, quantum information, quantum relative entropy

\section{Introduction}

The advances in the theory of quantum information [1] have gone far beyond any realization that can be even conceived at the present state of art. Production of many quantum bits entangled with each other, while disentangled with the environment is rather problematic.

Quantum error correction codes [2, 3] provide solutions for use in this struggle which are general, and not dependent on the actual realization of the qubits. It is on the other hand interesting to examine whether it is possible to protect quantum information against certain errors specific to the concrete physical systems.

There are several physical systems examined as candidates for representing quantum bits [4-8]. Our study concerns optical qubits, constituting one of the possibilities [9].

Optical qubits are embodied by modes of the electromagnetic field. The computational basis states $|0\rangle$ and |1) are identified by two orthogonal quantum states, e.g. two polarization states of a single spatial mode in the case of a polarization qubit, or the Fock states $|01\rangle$ and $|10\rangle$ of two field modes in the case of the dual-rail representation. Another possibility is to use a subspace of the Hilbert space of a single-mode light field spanned by a pair of sufficiently distant coherent states, which are approximately orthogonal. Such coherent state qubits have attracted much attention recently [10-12], including in quantum teleportation [13, 14], or theoretical realization of quantum circuits [15] based on these states.

On may chose an alternative computational basis for a coherent state qubit, namely two orthogonal optical Schrödinger cats: coherent superpositions of coherent states. These states were originally introduced by Dodonov et al [16] as 'even and odd coherent states'. They have per se the disadvantage of being highly non-classical, but the appearance of such states in the context of coherent state qubits is a natural consequence. The preparation of these states has already been investigated [17], but their detection, i.e. a projective measurement with Schrödinger cat eigenstates, is still an open question. Their manipulation - that is, realization of unitary transformations - is on the other hand a simple task using beam splitters.

The Schrödinger cats have the advantage of having more photons than few-photon Fock states; thus they are in a sense more 'macroscopic'. On the other hand, the essence of their nature is the phase-space interference between the constituent coherent states, which is extremely fragile against loss and environmental decoherence.

In this paper we demonstrate that it is possible to protect Schrödinger cats against the decohering effect of loss by applying a squeezing to their environment. The application of squeezing in order to lower noise is a prevalent technique 
in gravitational wave interferometry (see [18] and references therein). We do not address the technical question of realizing the squeezing of the environment, which depends on the particular implementation, but to give one example, a high $Q$ cavity containing a Schrödinger cat may be placed in another cavity sufficiently controlled to maintain its proper squeezing. For the case of transmission of qubits through optical fibres, one may think of the squeezing of the phononic modes of the media [19], since they are responsible for decoherence. We are here rather concerned with the theoretical estimation of whether squeezing is advantageous at all.

The decoherence behaviour of Schrödinger cats has already been investigated primarily in terms of phase-space distributions [20-25]. We adopt here a more informationtheoretical approach.

We examine the distinguishability via measurements of the two basis states of a qubit exposed to loss. This distinguishability is primarily a necessary and sufficient condition for classical communication applications. In a quantum information scenario the robustness in terms of distinguishability of two special orthogonal states is not a sufficient requirement for the usefulness in quantum computation. This can be regarded as a quantification of bitflip errors. Against these errors, a collection of error correcting codes have been developed; however, the circumstances are different during the computation process and the read-out process. The latter allows a different range of techniques.

For quantum computation in general, it is important to examine the decoherence behaviour of all the possible states of the qubit. When carrying out measurements to obtain the result of the computation, however, distinguishability of the computational basis states is in itself a relevant issue. Consider for instance the following scenario. We have a quantum logic network that solves a decision problem given a set of input qubits (such the one that realizes the Deutsch algorithm). The answer must be a simple 'yes' or 'no'; therefore the result can be encoded into the output qubits as either of the computational basis states. In order to convert this to classical information, a measurement is carried out with respect to the computational basis. The identification of the elements of the computational basis is therefore crucial to performing the read-out step. If we assume that the computation is deterministic, the resulting state may be obtained several times, and repeated measurements can be made. In certain cases we may assume that the quantum logic network is nearly ideal, but the detection in the readout step is lossy. In this case we arrive to the problem of distinguishing between the computational basis elements in a lossy environment via repeated measurements.

Our quantification of distinguishability relies on the concept of quantum relative entropy, which has also found important applications in the field of entanglement measures [26]. This quantity, though harder to calculate, has a more transparent operational meaning than simply the overlap of the states: it is directly related to the probability of faults in the identification of the given state. We develop a coherent state approach for its calculation in our scenario, which is applicable in different problems too.

Our main result is that appropriate squeezing of the environment (which is modelled here by a single bosonic mode) can significantly improve the distinguishability of the decohered Schrödinger cat states.

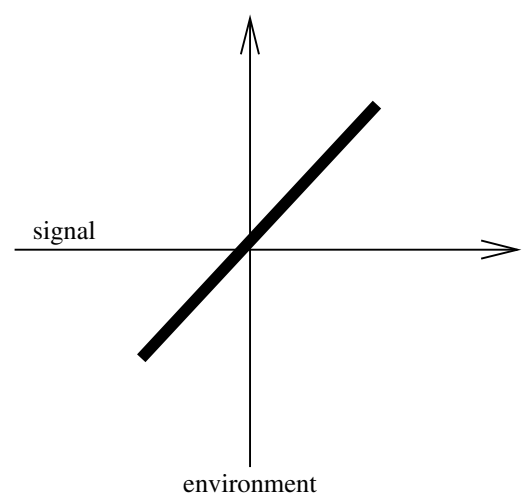

Figure 1. The model for loss. The signal mode interferes with an auxiliary 'environmental' mode on a nearly transparent beam splitter.

The paper is organized as follows. In section 2 we introduce the concepts required for our consideration. In section 3 the calculations of distinguishability are performed, and results are presented. In section 4 conclusions are drawn.

\section{Preliminaries}

In this section we describe Schrödinger cats, the simple model of loss applied in our consideration, and quantification of distinguishability of two quantum states.

\subsection{Schrödinger cats as a computational basis}

For representing a qubit there are two orthogonal states required, spanning the two-dimensional Hilbert space. In the case of travelling wave light fields, one may choose orthogonal Schrödinger cat states as the basis. If we denote a coherent state with amplitude $\alpha$ by $|\alpha\rangle$, in a general notation this basis can be written as

$$
|\alpha, s\rangle=\tilde{\mathcal{N}}_{s}^{-1} \sum_{\lambda= \pm 1} \lambda^{s}|\lambda \alpha\rangle \quad\left(=\tilde{\mathcal{N}}_{ \pm}^{-1}(|\alpha\rangle \pm|-\alpha\rangle)\right)
$$

where the normalization factor $\tilde{\mathcal{N}}_{s}^{2}=2\left[1+(-1)^{s} \mathrm{e}^{-2|\alpha|^{2}}\right]$. For $s=0,|\alpha, s\rangle$ is usually termed the 'even coherent state', + or the 'male' Schrödinger cat, and for $s=1$ it is usually the 'odd coherent state', - or 'female' Schrödinger cat. These states have the properties of being orthogonal and simple superpositions of coherent states. The use of coherent states as the basis motivates us to use the coherent state representation throughout our calculations.

\subsection{The model of loss}

A possible way of modelling loss is that depicted in figure 1 . The signal mode is interfering with an auxiliary mode, which represents a bosonic mode of the environment (a field mode or a phonon mode), usually assumed to be in a vacuum state. There are more sophisticated models for loss, derived e.g. from exact investigation of dispersing and absorbing media [27, 28]. In many of the cases, however, the simple model with one single beam splitter is satisfactory (cf, for instance, [29]).

In our consideration the state of the 'environment' mode is not necessarily vacuum. We shall squeeze our vacuum 
prior to mixing with the signal, which models physically the squeezing of the environmental mode. In the case of a squeezed environment the phase relations in the system become important, and the beam splitter parameters are written as $t=\mathrm{e}^{\mathrm{i} \varphi_{t}} \cos \tau$ and $r=\mathrm{e}^{\mathrm{i} \varphi_{r}} \sin \tau$, where the modulus of the transmittance $|t| \approx 1$ if we assume weak interaction with the environment.

\subsection{Quantification of the distinguishability of two quantum states}

To quantify the distinguishability of two quantum states, it is most appropriate to utilize the so-called quantum Sanov theorem (theorem 8 of [26]; see also [30] and [31]). The reasoning behind this is that we are considering the read-out problem of an outcome of a deterministic quantum calculation. Thus the output state may be reproduced as many times as we wish. We also assume that it is possible to construct a measurement that projects onto either of the possible outcomes, since the result of the computation corresponds to a classical bit. In this scenario, we have to distinguish between the two originally orthogonal quantum states.

The quantum Sanov theorem determines the probability of not distinguishing two quantum states described by the density matrices $\sigma$ and $\varrho$ after $n$ optimally chosen projective measurements. That is, the probability that after performing $n$ measurements on $n$ copies of a given quantum state $\sigma$, the results falsely yield that it was the state $\varrho$ is asymptotically

$$
P(\sigma \rightarrow \varrho)=\mathrm{e}^{-n S(\sigma \| \varrho)},
$$

where

$$
S(\sigma \| \varrho)=\operatorname{Tr} \sigma(\ln \sigma-\ln \varrho)
$$

is the quantum relative entropy. The higher the quantum relative entropy, the smaller the probability of a fault.

Since $S(\cdot \| \cdot)$ is not symmetric in its arguments, it might be easier to tell $\sigma$ from $\varrho$ than to tell $\varrho$ from $\sigma$. It is important to emphasize that quantum Sanov theorem gives a theoretical limit of distinguishability. This is not necessarily reached by an arbitrary measurement, but no optimized measurement can perform better.

\section{Decoherence and distinguishability of Schrödinger cats}

In this section, we shall apply the tools mentioned in section 2 to orthogonal Schrödinger cats $|\alpha, s\rangle$. First we shall calculate how the basis states are transformed by decoherence, then we shall develop a formula for expressing their quantum relative entropy.

\subsection{Decoherence of Schrödinger cats}

Consider the arrangement in figure 1. Let the state of the signal mode be one of the Schrödinger cats of equation (1). The environment mode is considered to be in a squeezed vacuum state, which can be expressed as a superposition of coherent states placed along a straight line in the phase space (i.e. in a one-dimensional coherent state representation) as [32-34]

$$
|S q, \zeta\rangle=\int_{\mathbb{R}} \mathrm{e}^{-\gamma x^{2}}\left|x \mathrm{e}^{\mathrm{i} \varphi}\right\rangle \mathrm{d} x
$$

Thus the whole system of the signal and environment modes is in the state (cf equations (1) and (4))

$$
\begin{aligned}
\left|\psi_{s}^{\text {in }}\right\rangle & =|\alpha, s\rangle|S q, \zeta\rangle \\
& =\mathcal{N}_{s}^{-1}(\gamma) \sum_{\lambda= \pm 1} \lambda^{s} \int_{\mathbb{R}} \mathrm{e}^{-\gamma x^{2}}|\lambda \alpha\rangle\left|x \mathrm{e}^{\mathrm{i} \varphi}\right\rangle \mathrm{d} x .
\end{aligned}
$$

The parameters of squeezing are $\gamma$ and $\varphi$ and are denoted according to Barnett and Radmore [35] as $\gamma=1 /(\exp (2|\zeta|)$ $-1)$ and $\varphi=\arg (\zeta) / 2+\pi$. The normalization factor is $\mathcal{N}_{s}(\gamma)=\tilde{\mathcal{N}}_{s} \mathcal{N}(\gamma)$, where $\mathcal{N}(\gamma)^{2}=\pi / \sqrt{\gamma^{2}+\gamma}$.

In the coherent state representation it is easy to describe the action of the beam splitter, as coherent states interfere simply as classical fields. The output amplitude for each coherent state pair is the sum of the transmitted and reflected amplitudes. To obtain the state remaining from the Schrödinger cat after the interference, this output density operator must be traced out in the environment mode. The resulting density matrix of the output modes of the beam splitter reads

$$
\begin{aligned}
\hat{\varrho}_{s}= & \mathcal{N}_{s}(\gamma)^{-2} \sum_{\lambda, v= \pm 1}(\lambda \nu)^{s} \int_{\mathbb{R}^{2}} \mathrm{e}^{-\gamma x^{2}} \mathrm{e}^{-\gamma y^{2}} \\
& \times\left\langle-v r^{*} \alpha+t^{*} \mathrm{e}^{\mathrm{i} \varphi} y \mid-\lambda r^{*} \alpha+t^{*} \mathrm{e}^{\mathrm{i} \varphi} x\right\rangle \\
& \times\left|\lambda t \alpha+r \mathrm{e}^{\mathrm{i} \varphi} x\right\rangle\left\langle v t \alpha+r \mathrm{e}^{\mathrm{i} \varphi} y\right| \mathrm{d} x \mathrm{~d} y
\end{aligned}
$$

where complex numbers $r$ and $t$ stand for the reflectivity and transmittivity of the beam splitter, including phase shifts. Our aim is now to calculate the relative entropy of the density matrix in equation (6).

\subsection{Calculation of relative entropies}

In order to calculate the relative entropy we take its power series around the identity operator. For arbitrary density matrices $\varrho$ and $\varrho^{\prime}$, this reads

$$
S\left(\hat{\varrho} \| \hat{\varrho}^{\prime}\right)=\sum_{n=1}^{\infty} \sum_{k=1}^{n}\left(\begin{array}{l}
n \\
k
\end{array}\right) \frac{(-1)^{k}}{n}\left(\operatorname{Tr} \hat{\varrho} \hat{\varrho}^{\prime k}-\operatorname{Tr} \hat{\varrho}^{k+1}\right) .
$$

For the decohered Schrödinger cat states $\varrho_{s}$ and $\varrho_{s^{\prime}}$ in the argument, the two traces in equation (6) produce a sum of Gaussian integrals:

$$
\begin{aligned}
& \operatorname{Tr}\left\{\hat{\rho}_{s} \hat{\rho}_{s^{\prime}}^{k}\right\}=\mathcal{N}_{s}(\gamma)^{-2} \mathcal{N}_{s^{\prime}}(\gamma)^{-2 k} \sum_{\lambda_{1}, v_{1}= \pm 1}\left(\lambda_{1} v_{1}\right)^{s} \\
& \quad \times \sum_{\lambda_{2}, v_{2}= \pm 1}\left(\lambda_{2} v_{2}\right)^{s^{\prime}} \ldots \sum_{\lambda_{k+1}, v_{k+1}= \pm 1}\left(\lambda_{k+1} v_{k+1}\right)^{s^{\prime}} \\
& \quad \times G_{k+1}\left(\lambda_{1}, v_{1}, \ldots, \lambda_{k+1}, v_{k+1}\right),
\end{aligned}
$$

where

$$
\begin{aligned}
& G_{k}\left(\left\{\lambda_{i}\right\},\left\{v_{i}\right\}\right)=\int_{\mathbb{R}^{2 k}} \prod_{l=1}^{k} \exp \left[-\gamma\left(x_{l}^{2}-y_{l}^{2}\right)\right] \\
& \quad \times\left\langle-v_{l} r^{*} \alpha+t^{*} \mathrm{e}^{\mathrm{i} \varphi} y_{l} \mid-\lambda_{l} r^{*} \alpha+t^{*} \mathrm{e}^{\mathrm{i} \varphi} x_{l}\right\rangle \\
& \quad \times\left\langle v_{l} t \alpha+r \mathrm{e}^{\mathrm{i} \varphi} y_{l} \mid \lambda_{l+1} t \alpha+r \mathrm{e}^{\mathrm{i} \varphi} x_{l+1}\right\rangle \mathrm{d}^{k} x \mathrm{~d}^{k} y .
\end{aligned}
$$

(Modulo $k$ is assumed for the indices.) After expanding the coherent state products and introducing

$$
\begin{aligned}
(\boldsymbol{A})_{l m} & =-\left(\gamma+\frac{1}{2}\right) \delta_{l m}+|t|^{2} \theta(k-m) \delta_{l, m+k} \\
& +|r|^{2} \theta(k-m) \delta_{l,[m-1]_{k}+k} \\
(\boldsymbol{b})_{l} & =\theta_{k-l}\left(\lambda_{[l-1]_{k}}-\lambda_{l}\right) r t^{*} \alpha^{*} \mathrm{e}^{\mathrm{i} \varphi} \\
& +\theta_{l-(k+1)}\left(\lambda_{k+[l-k+1]_{k}}-\lambda_{l}\right) r^{*} t \alpha \mathrm{e}^{-\mathrm{i} \varphi}, \\
c= & \sum_{l=1}^{k}\left(\lambda_{l} \lambda_{[l+k+1]_{k}+k}|r|^{2}+\lambda_{l} \lambda_{l+k}|t|^{2}\right)|\alpha|^{2}-k|\alpha|^{2},
\end{aligned}
$$




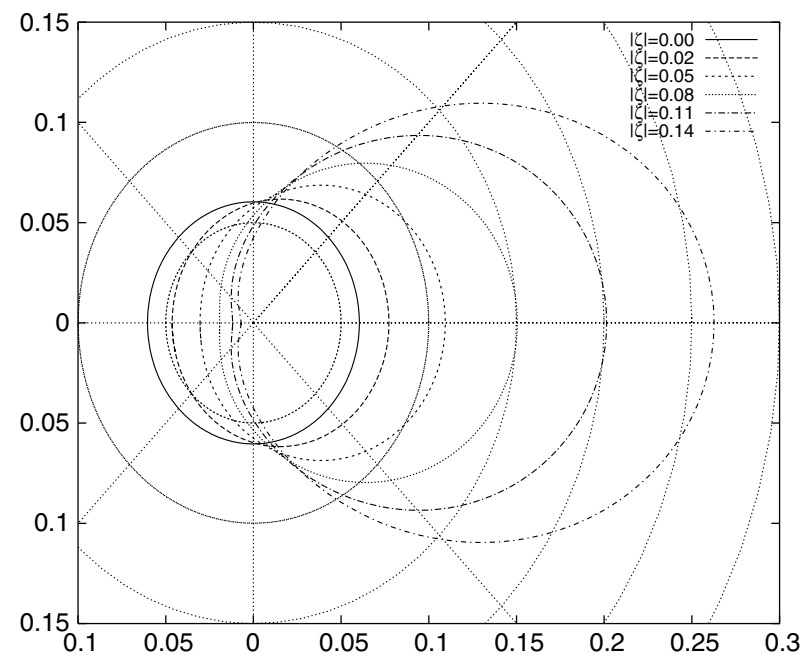

Figure 2. A polar plot of the dependence of the relative entropy $S(+\|-)$ on the relative phase-space orientation angle $\phi$ of equation (19) of the cat state and the squeezing of the environment for different magnitudes of squeezing. The beam splitter transmittivity is $t=0.95 ;|\alpha|=3$.

with

$$
\begin{aligned}
(\boldsymbol{x})_{i} & := \begin{cases}x_{i}, & \text { when } i \leqslant k, \\
y_{i-k}, & \text { otherwise, }\end{cases} \\
(\boldsymbol{\lambda})_{i} & := \begin{cases}v_{i}, & \text { when } i \leqslant k, \\
\lambda_{i-k}, & \text { otherwise, }\end{cases} \\
\theta_{i} & := \begin{cases}1, & \text { if } i \geqslant 0, \\
0, & \text { otherwise, }\end{cases}
\end{aligned}
$$

and $\boldsymbol{Q}=\frac{1}{2}\left(\boldsymbol{A}+\boldsymbol{A}^{\mathrm{T}}\right), G_{k}$ can be expressed explicitly:

$$
\begin{aligned}
G_{k}(\boldsymbol{\lambda}) & =\int_{\mathbb{R}^{2 k}} \exp \left[\boldsymbol{x}^{\mathrm{T}} \boldsymbol{A} \boldsymbol{x}+\boldsymbol{b}^{\mathrm{T}} \boldsymbol{x}+c\right] \mathrm{d}^{2 k} \boldsymbol{x} \\
& =\frac{\pi^{k}}{\sqrt{\operatorname{det} \boldsymbol{Q}}} \mathrm{e}^{-\frac{1}{4} \boldsymbol{b}^{\mathrm{T}} \boldsymbol{Q}^{-1} \boldsymbol{b}+c} .
\end{aligned}
$$

From equations (7), (8) and (16) we obtain the relative entropy of two decohered Schrödinger cat states $\varrho_{s}$ and $\varrho_{s^{\prime}}$ as

$$
S\left(\hat{\rho}_{s} \| \hat{\rho}_{s^{\prime}}\right)=\sum_{n=1}^{\infty} \frac{1}{n} \sum_{k=1}^{n}\left(\begin{array}{l}
n \\
k
\end{array}\right) \frac{(-1)^{k} \pi^{k+1}}{\sqrt{\operatorname{det} Q}} F_{k},
$$

where

$$
\begin{aligned}
F_{k}= & \sum_{\lambda \in\{ \pm 1\}^{2 k+2}} \mathcal{N}_{s}(\gamma)^{-2}\left(\lambda_{k+1} \lambda_{2 k+2}\right)^{s} \\
& \times\left[\mathcal{N}_{s^{\prime}}(\gamma)^{-2 k} \prod_{l=1}^{k}\left(\lambda_{l} \lambda_{k+1+l}\right)^{s^{\prime}}-\mathcal{N}_{s}(\gamma)^{-2 k} \prod_{l=1}^{k}\left(\lambda_{l} \lambda_{k+1+l}\right)^{s}\right] \\
& \times \mathrm{e}^{-\frac{1}{4} b^{\mathrm{T}} Q^{-1} b+c} .
\end{aligned}
$$

The series expansion in equation (17) is convergent in norm, and though its convergence is not very fast, it can be evaluated numerically. The data plots presented in this paper result from this evaluation.

We remark that for $|\alpha| \ll 1$, the convergence of the series in equation (17) is slow. Our method is suitable for larger values of $|\alpha|$, which is the physically interesting case. Therefore the $|\alpha|$ scales of the figures start at 1 .

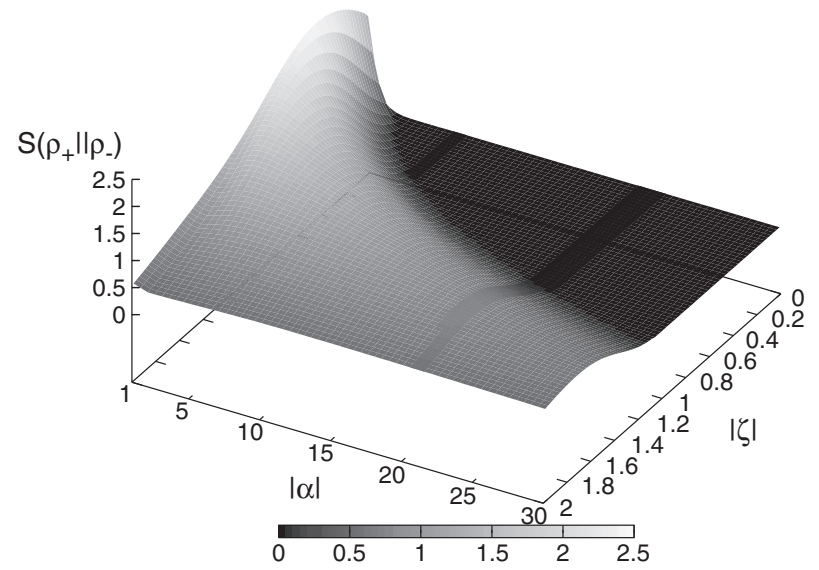

Figure 3. Contour level plots of the maximally improved entropy $S(+\|-)$ versus the magnitude $|\zeta|$ of squeezing and the magnitude of the $|\alpha|$ parameter of the coherent state constituting the cats. The beam splitter transmittivity is $t=0.95$.

\subsection{Results}

In what follows, the two relative entropies in the argument will be denoted by $S(+\|-)$ and $S(-\|+)$ respectively. We chose the beam splitter transmittivity to be 0.95 . For smaller transmittivity (i.e. higher loss), the behaviour of the entropies is similar, but the actual values are smaller.

The calculations confirm a fact that can be expected for symmetry reasons: that, regarding the angular parameters, the relative entropy depends on the following combination:

$$
\phi=\arg (t)+\arg (r)+\arg (\alpha)+\frac{1}{2} \arg (\zeta) .
$$

This means the parameters on the left-hand side, namely the phase shift imparted by the beam splitter to the transmitted and reflected beams, the polar angle of the coherent state parameter, and the direction of the squeezing appear in the above combination only. As in the output state the input mode appears as transmitted, while the environment mode appears as reflected, $\phi$ describes the direction of the squeezing relative to the orientation of the Schrödinger cats. The dependence on $\phi$ of the relative entropy $S(+\|-)$ is plotted in figure 2 . The relative entropy $S(-\|+)$ shows the very same behaviour. From this we conclude, for a given pair of Schrödinger cats, that squeezing in the appropriate direction increases both relative entropies above the value corresponding to the vacuum environment state $(\zeta=0)$. On the other hand, an orthogonal direction of squeezing (notice the $1 / 2$ factor in equation (19)) can even worsen the situation. This is typical for squeezing phenomena. In what follows, we treat only the case of maximal improvement, i.e. $\phi=0$.

In figure 3 we have plotted the maximally improved entropy $S(+\|-)$ versus the magnitude of squeezing $|\zeta|$ and the magnitude of the $|\alpha|$ parameter of the coherent states constituting the cat. Note that $|\alpha|$ is the distance in phase space between the two superposed coherent states. Large values correspond to the quantum superposition of really macroscopic states.

The entropy $S(+\|-)$ is so similar to $S(-\|+)$ that the difference would not be visible in a plot such as figure 3 . To illustrate this, in figure 4 we have plotted both entropies as a 


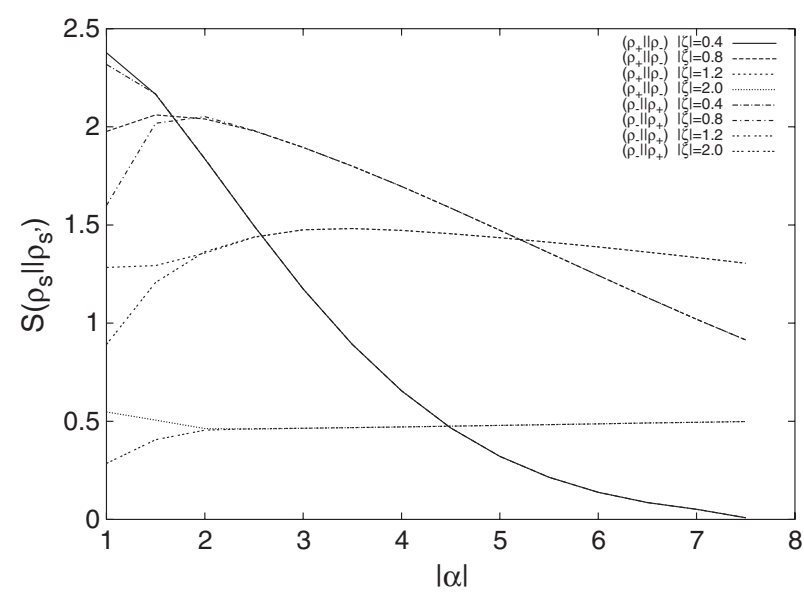

Figure 4. Plots of both relative entropies $S(+\|-)$ and $S(-\|+)$ versus the magnitude of the parameter $\alpha$ of the cats, for different magnitudes $|\zeta|$ of the squeezing. The beam splitter transmittivity is $t=0.95$.

function of $|\alpha|$. It can be seen that the difference between the two entropies is relevant mainly for small $|\alpha|$ values, which is the less interesting case. This is somewhat to be expected, as for larger $|\alpha|$ values the two states are in a sense more similar, which is apparent e.g. from the shape of their Wigner functions. Thus the fault probability can be expected to be symmetric in this case.

Returning to figure 3, we find that for a given not too large $|\alpha|$, there is an optimal magnitude of squeezing, for which the improvement in the distinguishability of the two cats is the highest. For higher $|\alpha|$ values - that is, for superpositions of more macroscopic coherent states-more squeezing is needed. This is natural as the two cats differ only in the well-known phase-space interference pattern at the origin of the phase space, which is extremely fragile for macroscopic states. It is also visible that for high $|\alpha|$ values, squeezing cannot provide a relevant improvement against decoherence.

It is remarkable that for $|\alpha|=3$, where the achievable improvement is rather significant, there are about ten photons present on average in the two Schrödinger cats. This means that these states can play the role of a computational basis with relatively high photon number, in comparison with the polarization or dual-rail representation of qubits.

\section{Conclusions}

We have examined the behaviour of two orthogonal Schrödinger cat states against loss, from the point of view of distinguishability. The main concern was to provide an example where a certain representation of qubits can be protected against loss in a way specific to the concrete physical realization. Distinguishability was quantified via the quantum relative entropy, in the spirit of the quantum Sanov theorem.

We have studied the possible improvement achievable by squeezing the environment mode. We have evaluated the relative entropies using a power series, derived in the lowdimensional coherent state representation.

We have found that for not too macroscopic superpositions of coherent states, an improvement of the distinguishability can be achieved by appropriately chosen squeezing. Both the phase and magnitude of the squeezing are optimal for a given parameter of the coherent state constituting the Schrödinger cats.

In general, it would be worth finding an easy-to-calculate quantitative feature for a couple of quantum states of physical systems, which describes their applicability for representing qubits. It could then be calculated instead of, for instance, the relative entropy.

\section{Acknowledgments}

This work was supported by the Research Fund of Hungary (OTKA) under contracts No T034484, T043079 and T043287. We thank the Computer Services Centre, Faculty of Science, University of Pécs, for providing computer resources.

\section{References}

[1] Nielsen M A and Chuang I L 2000 Quantum Computation and Quantum Information (Cambridge: Cambridge University Press)

[2] Shor P 1995 Phys. Rev. A 42 R2493

[3] Knill E, Laflamme R and Viola L 2000 Phys. Rev. Lett. 84 2525

[4] Gershenfeld N A and Chuang I L 1997 Science 275350

[5] Sasura M and Buzek V 2002 J. Mod. Opt. 491593

[6] Leibfried D, DeMarco B, Meyer V, Lucas D, Barrett M, Britton J, Itano W M, Jelenkovic B, Langer C, Rosenband T and Wineland D J 2003 Nature $\mathbf{4 2 2} 412$

[7] Shahriar M S, Hemmer P R, Lloyd S, Bhatia P S and Craig A E 2002 Phys. Rev. A 66032301

[8] Chiorescu I, Nakamura Y, Harmans C J P M and Mooij J E 2003 Science 2991869

[9] Knill E, Laflamme R and Milburn G J 2001 Nature 40946

[10] van Enk S J 2003 Phys. Rev. Lett. 91017902

[11] Jeong H, Kim M S and Lee J 2001 Phys. Rev. A 64052308

[12] Wang X 2001 Phys. Rev. A 64022302

[13] van Enk S J and Hirota O 2001 Phys. Rev. A 64022313

[14] Nguyen B A 2003 Phys. Rev. A 68022321

[15] Jeong H and Kim M S 2002 Phys. Rev. A 65042305

[16] Dodonov V V, Malkin I A and Man'ko V I 1974 Physica 72 597

[17] Yurke B and Stoler D 1986 Phys. Rev. Lett. 5713

[18] Kimble H J, Levin Y, Matsko A B, Thorne K S and Vyatchanin S P 2002 Phys. Rev. D 65022002

[19] Garrett G A, Rojo A G, Sood A K, Whitaker J F and Merlin R 1997 Science 2751638

[20] Janszky J, Petak A, Sibilia C, Bertolotti M and Adam P 1995 Quantum Semiclass. Opt. 7145

[21] Filip R and Perina J 2001 J. Opt. B: Quantum Semiclass. Opt. 321

[22] El-Orany F A A, Perina J, Perinova V and Abdalla M S 2002 J. Opt. B: Quantum Semiclass. Opt. $4 \mathrm{~S} 153$

[23] El-Orany F A A, Perina J, Perinova V and Abdalla M S 2003 Eur. Phys. J. D 22141

[24] El-Orany F A A 2002 Phys. Rev. A 65043814

[25] Foldi P, Benedict M G, Czirjak A and Molnar B 2003 Phys. Rev. A 67032104

[26] Vedral V and Plenio M B 1998 Phys. Rev. A 571619

[27] Knoll L, Scheel S, Schmidt E, Welsch D G and Chizhov A V 1999 Phys. Rev. A 594716

[28] Scheel S, Knoll L, Opatrny T and Welsch D G 2000 Phys. Rev A 62043803 
Distinguishing Schrödinger cats in a lossy environment

[29] Vukics A, Janszky J and Kobayashi T 2002 Phys. Rev. A 66 023809

[30] Hiai F and Petz D 1991 Commun. Math. Phys. 14399

[31] Vedral V 2002 Rev. Mod. Phys. 74197

[32] Janszky J and Vinogradov A V 1990 Phys. Rev. Lett. 64 2771
[33] Janszky J, Koniorczyk M and Gábris A 2001 Phys. Rev. A 64 034302

[34] Janszky J, Gábris A, Koniorczyk M, Vukics A and Adam P 2001 Fortschr. Phys. 49993

[35] Radmore P M and Barnett S M 1997 Methods in Theoretical Quantum Optics (Oxford: Oxford University Press) 